\title{
Brugada Syndrome, QRS-Fragmentation, Wavy Triple Sign (Yasser's sign) with Myocardial Ischemia in Terminal Metastatic Prostatic Carcinoma
}

\author{
Yasser Mohammed Hassanain Elsayed* \\ Critical Care Unit, Kafr El-Bateekh Central Hospital Egypt
}

\begin{abstract}
Rationale: Prostatic carcinoma is the most frequently detected malignancy and the sixth leading cause of tumor mortality in men. Gleason grade is one of the most important prognosticators in prostate carcinoma. Brugada syndrome (BrS) is a genetic disorder with abnormal electrical activity within the heart. The syndrome carries a higher risk for ventricular fibrillation and sudden death. The presence of fQRS can be yielding regional conduction decelerating such as ischemia, scar, fibrosis, myofiber disarray, inflammation, and microvascular abnormality. Wavy triple an electrocardiographic sign (Yasser's Sign) is a novel diagnostic electrocardiographic sign was prescribed in hypocalcemia.
\end{abstract}

Patient concerns: A 70-year-old married, worker, smoker, Egyptian, male patient was admitted to the intensive care unit with metastatic prostatic carcinoma, suspected pulmonary embolism, hepatorenal impairment involvement, and numerous electrocardiographic signs.

Diagnosis: Metastatic prostatic carcinoma with suspected pulmonary embolism and hepatorenal impairment involvement.

Interventions: Electrocardiography, oxygenation, and CT pulmonary angiogram.

Outcomes: Electrocardiographic and clinical stabilization was the only result.

Lessons: The presence of elderly, myocardial ischemia, heavy cigarette smoking, Wavy triple sign or (Yasser's sign), hepatorenal impairment, and QRS-fragmentation in metastatic prostatic carcinoma are a constellation of serious risk factors. The De-Winter sign is an equivalent of acute myocardial infarction with considering of up-sloping ST-segment depression.

KEYWORDS: Brugada syndrome; QRS-Fragmentation; Wavy triple sign; Ischemic heart disease; Metastatic prostatic carcinoma

ABBREVIATIONS: ECG: Electrocardiogram; ICU: Intensive Care Unit; 02: Oxygen; SGOT: Serum Glutamic-Oxaloacetic Transaminase; SGPT: Serum Glutamic-Pyruvic Transaminase; VR: Ventricular Rate

\section{INTRODUCTION}

Worldwide, prostatic carcinoma is the most frequently detected malignancy and the sixth leading cause of tumor mortality in men [1]. Fortunately, most prostatic carcinomas are slowly growing, low-grade, and not highly aggressive. If there is carcinoma in-situ, it is considered localized and potentially curable. But if cancer had spread elsewhere in the body, then, analgesia, bisphosphonates, ligand inhibitors, hormonal therapy, chemotherapy, radiopharmaceuticals, immunotherapy, focused radiation, and targeted therapies will be optional therapies.
Quick Response Code:

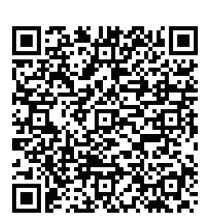

Address for correspondence: Elsayed YMH, Critical Care Unit, Kafr El-Bateekh Central Hospital, Damietta Health Affairs, Egyptian Ministry of Health (MOH), Damietta, Egypt

Received: October 15, 2021

Published: November 12, 2021

How to cite this article: Yasser MHE. Brugada Syndrome, QRS-Fragmentation, Wavy Triple Sign (Yasser's sign) with Myocardial Ischemia in Terminal Metastatic Prostatic Carcinoma. 2021- 3(6) OAJBS.ID.000346. DOI: $10.38125 /$ OAJBS.000346 
Outcomes are commonly based on age, co-morbid disorders, tumor histopathology, and the extent of tumor. Brugada syndrome $(\mathrm{BrS})$ is a genetic disorder with abnormal electrical activity within the heart. The syndrome carries a higher risk for ventricular fibrillation (VF) and sudden cardiac death (SCD) in a structurally normal heart (SHD) [2]. Three classes were described; Coved type (class I) has a coved type of ST-segment elevation with $\geq 2 \mathrm{~mm}(0.2 \mathrm{mV})$ J-point elevation and a gradually descending ST-segment followed by a negative T-wave [2]. The term 'fragmentation of the QRS-complex" or "fragmented QRS" (fQRS) point to the existence of high-frequency potentials (spikes) in the QRS-complex [3]. The presence of fQRS can be induced by any condition interrupting with the normally homogeneous depolarisation status in the myocardium and yielding regional conduction decelerating such as ischemia, scar, fibrosis, myofiber disarray, inflammation, and microvascular abnormality. Das et al. revealed that there was a good correlation between fQRS and the presence of myocardial scar in patients with ischemic heart disease (IHD) that appeared by single-photon emission tomography (SPET) [4]. Wavy triple an electrocardiographic sign (Yasser's sign) is a new specific diagnostic sign seen in $97.3 \%$ of the cases of hypocalcemia. Wavy triple an electrocardiographic sign can be used as a therapeutic guide in the cases of hypocalcemia [5]. Patients with up-sloping ST-segment depression had a comparable rate of in-hospital revascularization versus those with non-upsloping ST-segment depression. This is suggesting that up-sloping ST-segment depression should be recognized as a manifestation of ischemia in NSTEMI [6].

\section{CASE PRESENTATION}

A 70-year-old married, worker, smoker, Egyptian, male patient was admitted to the intensive care unit (ICU) with tachypnea and palpitations. Dizziness, profuse sweating, generalized body aches, and fatigue were the associated symptoms. The patient is a currently heavy smoker (at least 20 cigarettes for about 25 years). He gave a history of prostatic carcinoma to his brother. Upon general physical examination, generally, the patient was irritable, anxious, and distressed, with a regular rapid heart rate of $150 \mathrm{bpm}$, blood pressure of $90 / 70 \mathrm{mmHg}$, respiratory rate of $28 \mathrm{bpm}$, the temperature of $37.2{ }^{\circ} \mathrm{C}$, and pulse oximeter of 02 saturation of $94 \%$. No more relevant clinical data were noted during the clinical examination. Urgent initial ECG tracing was done on the presentation in the ICU showing a Wavy triple sign appears in III, V2, and V3 leads, up-sloping ST-segments elevation, coving STsegments elevation of Brugada syndrome, and QRS-fragmentations (Figure 1).

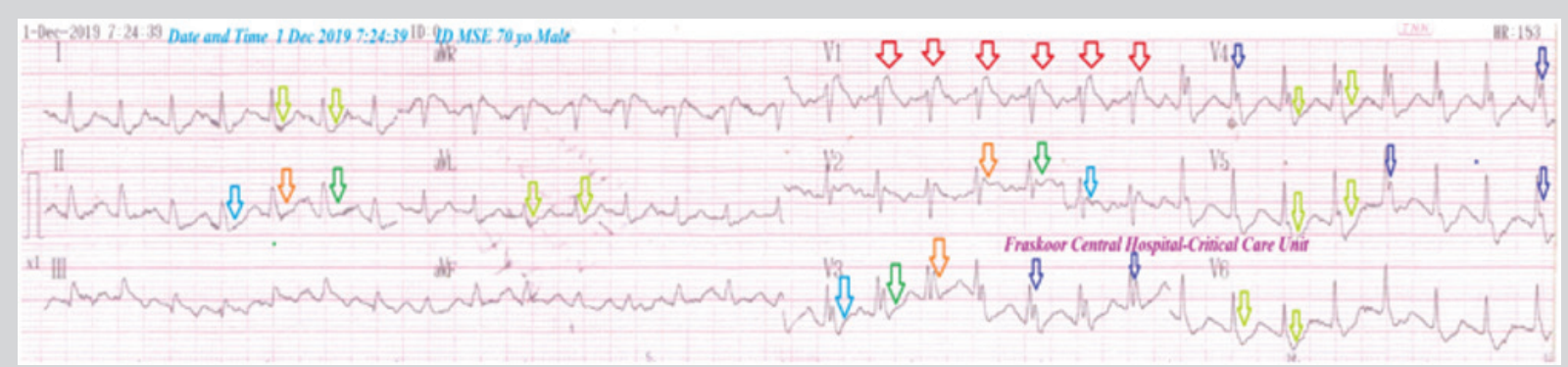

Figure 1: An initial ECG tracing was done on the presentation in the ICU showing a Wavy triple sign appearing in III, V2, and V3 leads (Yasser's sign; green, red, and blue arrows), up-sloping ST-segments elevation (lime arrows). Coving ST-segments elevation and partial RBBB in V1-2 with a coved morphology the (Brugada sign; red arrows), and QRS-fragmentation (dark blue arrows).

The patient was conservatively treated 02 inhalation (100\%, by nasal cannula, $5 \mathrm{~L} / \mathrm{min}$ ), cefotaxime; (1000 mg IV every 8hours), IV of ampicillin with sulbactam vial (1.5gm, TDS, for 3 days), paracetamol (500mg IV every 8 hours as needed), SC enoxaparin 40mg twice daily), aspirin tablet (75mg, once daily), and clopidogrel tablet (75mg, once daily. The complete blood count (CBC); Hb was $9.7 \mathrm{~g} / \mathrm{dl}$, RBCs; 3.90*103/mm3, WBCs; 18.4*103/mm3 (Neutrophils; $48 \%$, Lymphocytes: 44\%, Monocytes; 7\%, Eosinophils; $1 \%$ and Basophils $0 \%)$, Platelets; $366^{*} 103 / \mathrm{mm} 3$. Serum creatinine was high $(3.1 \mathrm{mg} /$ dl) and blood urea was high $(156 \mathrm{mg} / \mathrm{dl})$. RBS was normal $(149 \mathrm{mg} /$ dl). Ionized calcium was slightly low $(0.73 \mathrm{mmol} / \mathrm{L})$, plasma sodium was high $(147 \mathrm{mmol} / \mathrm{L})$, and serum potassium was normal (5.2mmol/L).SGPT was high (55U/L), SGOT was high (61U/L). Total bilirubin was high $(1.7 \mathrm{mg} / \mathrm{dl})$. D-dimer was normal $(1234 \mathrm{ng} / \mathrm{ml})$. The troponin test was positive (43ng/L). ABG showed respiratory alkalosis. MRI-targeted biopsy with ultrasound and PSA were used in the initial diagnosis and follow-up. Metastatic prostatic carcinoma with suspected pulmonary embolism and hepatorenal impairment involvement was the most probable current diagnosis. The patient was discharged on the third day after general and clinical stabilization. The patient was continued: aspirin tablet (75mg, once daily), and clopidogrel tablet (75mg, once daily), and warfarin tablet (5mg, once daily). The patient was advised for cardiovascular and oncological diseases follow-up.

\section{COMMENTS}

\section{Overview}

A 70-year-old married, worker, smoker, Egyptian, male patient was admitted to the intensive care unit with metastatic prostatic carcinoma, suspected pulmonary embolism, hepatorenal impairment involvement, and numerous ECG signs.

\section{Primary Objective}

For my case study was the presence of a patient who presented with metastatic prostatic carcinoma with suspected acute pulmonary embolism and hepatorenal impairment involvement in the ICU.

\section{Secondary Objective}

For my case study was the question of; How did you manage the case at home? The presence of tachypnea, sinus tachycardia, and elevated D-dimer will strengthen the acute pulmonary embolism diagnosis. A CT pulmonary angiogram (or CTPA) is contraindicated in renal impairment. An elevated troponin can be interpreted either by associated myocardial ischemia or as a poor prognostic point in the acute pulmonary embolism. The presence of fragmentation of the QRS-complex is considered another risk. The existence of 
respiratory alkalosis is the indicator for the current Wavy triple sign or (Yasser's sign) of hypocalcemia. Right bundle branch block (RBBB) and De-Winter sign are considered in the differential diagnosis. RBBB is mostly restricted to V1-V2 but the De-Winter sign is an equivalent of anterior ST-segment elevation myocardial infarction (STEMI). Up sloping ST-segment depression $>1 \mathrm{~mm}$ at the J-point in the precordial leads [7].

\section{CONCLUSION AND RECOMMENDATIONS}

- The presence of elderly, myocardial ischemia, heavy cigarette smoking, Wavy triple sign or (Yasser's sign), hepatorenal impairment, and QRS-fragmentation in metastatic prostatic carcinoma are a constellation of serious risk factors.

- The De-Winter sign is an equivalent of acute myocardial infarction with considering of up-sloping ST-segment depression.

\section{REFERENCES}

1. Leslie SW, Soon-Sutton TL, Sajjad H, Siref LE (2021) Prostate cancer. In: StatPearls Publishing USA.
2. Elsayed YMH (2019) An electrocardiographic triple rhythm of sinus arrhythmia, brugada syndrome, and early repolarization with a diverse outcome. WW Med 1(9): 303-306.

3. Brohet C (2019) Fragmentation of the QRS complex: the latest electrocardiographic craze? Acta Cardiologica 74(3): 185-187.

4. Das MK, Khan B, Jacob S, Kumar A, Mahenthiran J (2006) Significance of a fragmented QRS complex versus a $Q$ wave in patients with coronary artery disease. Circulation 113(21): 2495-2501.

5. Elsayed YMH (2019) Wavy triple an electrocardiographic sign (Yasser Sign) in hypocalcemia. A novel diagnostic sign; retrospective observational study. EC Emergency Medicine and Critical Care (ECEC). $3(2): 1-2$.

6. Misumida N, Kobayashi A, Schweitzer P, Kanei Y (2015) Prevalence and clinical significance of up-sloping ST-segment depression in patients with non-ST-segment elevation myocardial infarction. Cardiol Res 6(45): 306-310.

7. Elsayed YMH (2019) De winter sign as a precursor to acute myocardial infarction and post-streptokinase dramatic response; A case report. IJRSMHS 4(10): 17-20. 